\title{
Grenzwertig im Dazwischen. Liminalität als DenkRaum
}

Schlüsselwörter: Kulturtheorie, Kulturwissenschaft, Literaturwissenschaft, Liminalität, literarische Anthropologie, Grenzen, Überschreitung, Gespenst, Vampir, Dritter Raum, Schwellen

DOI: $10.4312 /$ ars.13.2.26-39

Er hält sich abwechselnd auf dem Dachboden, im Treppenhaus, auf den Gängen, im Flur auf. [...] "Wie heißt du denn?" fragt man ihn. "Odradek", sagt er. "Und wo wohnst du?" "Unbestimmter Wohnsitz", sagt er und lacht; es ist aber nur ein Lachen, wie man es ohne Lungen hervorbringen kann. (Kafka, 1994, 223)

Kafkas mysteriöses Wesen Odradek, dessen Name ebenso wenig festzumachen ist wie sein Aussehen oder Wohnort, ist der Paradefall einer 'Existenz'(?), die "betwixt and between" ist: so lautet die berühmte Formel, die der schottisch-amerikanische Anthropologe Victor Turner (1920-1983) für sein Modell der Liminalität geprägt hat (2005, 95). Dabei handelt es sich ursprünglich um eine räumliche Denkfigur: "Liminal" ist ein Zwischenraum zwischen zwei Zuständen, die sich auch als Gebiete im buchstäblichen oder übertragenen Sinn verstehen lassen - wobei im Zentrum dieses Vorstellungsraums ein Konzept der Grenze (des limen) steht, die diese nicht als körperlose Linie, sondern als Schwelle, ja als Pufferzone zwischen zwei Sphären sieht: ein Durchgangsbereich, in dem man innehalten, aber auch zwischen diskursiven oder realen Fronten gefangen sein kann. 'Liminalität' kann also auch als art of becoming verstanden werden (etwa bei einem Initiationsritus zwischen Kindheit und Erwachsenenalter), ja im übertragenen Sinn die Utopie des eingeschlossenen Dritten darstellen, die dazu dient, die Falltüren einer binären Entweder-Oder-Logik zu überbrücken; es kann aber auch der unbefriedigende Zustand eines Nicht-mehr-und-Noch-nicht in einem Niemandsland sein, der die sich Aufhaltenden aus beiden an einander angrenzenden Räumen ausschließt - so wie etwa jene Passagiere, die aufgrund eines Visaproblems scheinbar endlos im Transitraum eines Flughafens festgehalten werden.

Im Folgenden soll nun in losem Anschluss an einige Gedanken meines Buches Am Rande zur Dynamik von Zentrum/Peripherie (Ruthner, 2004, Kap. 1) sowie eines Aufsatzes zur fantastischen Literatur (Ruthner, 2010 u. 2012) das Potenzial des Konzepts 
'Liminalität' für die humanities provisorisch ausgelotet werden; als Ausgangspunkt dient neben dem erwähnten Konzept der Grenze die damit verbundene Vorstellung der Überschreitung bzw. Transgression. Zum Abschluss wird dann nach dem heuristischen (Grenz)Wert gefragt, wobei sich der vorliegende Beitrag als erster Aufriss eines umfangreichen Publikationsprojekts versteht, ${ }^{1}$ das die in unterschiedlichen Kontexten entstehende Theoriebildung mit einbeziehen wird (so etwa Aguirre, Sutton, 2000; Lachmayer, Plica, 2003; Geisenhanslüke, Mein, 2008; Achilles u. a., 2012; Klapcsik, 2012; Krüger, 2018; u. v. a.).

\section{Grenzen}

Die Tendenz zur Aufteilung des Raumes unter Menschen(gruppen), d. h. sein 'Besitz' und seine Bewohnung, scheint ein unabdingbarer Teil unserer Natur zu sein, mit dem sich das Territorialverhalten aller höheren Säugetiere als evolutionäres Erbe auch in der Grundlage menschlicher Kultur fortsetzen mag. Der Irrtum besteht indes darin, aufgrund dieser biologischen Veranlagung alle damit einhergehenden Phänomene der Territorialität zu naturalisieren.

Dies hat schon Georg Simmel zu Beginn des 20. Jahrhunderts in seiner Soziologie des Raums erkannt; eine "Qualität des Raumes, die auf die gesellschaftlichen Wechselwirkungen wesentlich einwirkt", so schreibt er, liege darin,

daß sich der Raum für unsere praktische Ausnutzung in Stücke zerlegt, die als Einheiten gelten und - als Ursache wie als Wirkung hiervon - von Grenzen eingerahmt sind. [...] Der Rahmen, die in sich zurücklaufende Grenze eines Gebildes, hat für die soziale Gruppe sehr ähnliche Bedeutung wie für ein Kunstwerk. (Simmel, 1995, 138)

Interessant ist hier die Engführung von geografischer bzw. politischer Grenze und ästhetischem Rahmen, wobei Simmel auf deren beider Gemachtheit abhebt und mit dem Vorurteil, es gäbe 'natürliche' Begrenzungen (wie z. B. Flüsse) aufräumt: "Der Begriff der Grenze" sei "in allen Verhältnissen von Menschen untereinander äußerst wichtig", zumal er (soziale) "Sphären" schaffe - wenngleich "sein Sinn nicht immer ein soziologischer ist" (1995, 140). So sei die Grenze "nicht eine räumliche Tatsache mit soziologischen Wirkungen, sondern eine soziologische Tatsache, die sich räumlich formt" (141); damit bekommt sie nicht nur einen sozialregulatorischen, sondern auch einen ästhetischen Wert, wie dies ein Sammelband zur neuen Sub- und Transdisziplin der sog. "Border Aesthetics" programmatisch formuliert hat (Rosello, Wolfe, 2017, 6ff.).

1 Geplant ist ein substanzieller Sammelband zum Thema Liminality and Literature bei Cambridge University Press in Zusammenarbeit mit der Limen-Forschungsgruppe rund um den spanischen Anglisten Manuel Aguirre (Universidad Autónoma de Madrid). 
Die Grenze selbst ist damit streng genommen ein "Nicht-Ort" (Augé, 2008, 63), wie das auch Francesco Magris, Sohn des bekannten Triestiner Autors, als Grenzlandbewohner quasi-philosophisch formuliert hat $(2019,34)$; genauso ist sie aber nicht nur eine Trennlinie, sondern meist eine Zone - "a borderscape" (Rosello, Wolfe, 2017, 8ff.) - die weder mit dem einen oder dem anderen Raum ident ist. Hiergegen hat freilich schon Walter Benjamin opponiert, wenn er schreibt:

Die Schwelle ist ganz scharf von der Grenze zu scheiden. Schwelle ist eine Zone. Wandel, Übergang, Fluten liegen im Worte 'schwellen' [...]. Andererseits ist notwendig, den unmittelbaren tektonischen und zeremonialen Zusammenhang festzustellen, der das Wort zu seiner Bedeutung gebracht. $(1983,618)$

Funktional differenziert der erwähnte Sammelband Border Aesthetics wie folgt: "Bordering is ordering, othering and negotiating difference." (Rosello, Wolfe, 2017, 12) Magris schreibt: "Die Grenze kann mal beschützen, mal gefangen nehmen, sie verschließt sich in sich selbst oder dehnt sich aus, um sich das einzuverleiben, was außerhalb von ihr liegt." $(2019,34)$ Paradoxerweise trennen aber Grenzen nicht nur, sondern sie verbinden auch (Rosello, Wolfe, 2017, 11; Bhabha, 2016, 80f.) Sie können "zur Barriere, zur Schranke oder [...] zum Filter oder zur Membran werden." (Donec, 2014, 30)

Mit der Möglichkeit der Gliederung des Raums durch Grenzen in Territorien ergibt sich nämlich auch die virtuelle Möglichkeit zu deren Überschreitung, sei es auf Einladung (Gastfreundschaft ${ }^{2}$ ) oder feindselig, als Intrusion, Invasion, Transgression, aber auch als Überbrückung und Verbindung.

\section{2 Überschreitung}

Dieser Begriff soll hier das Übertreten einer ontologischen, sozialen, kulturellen oder symbolischen Grenze bedeuten - also nicht unbedingt in dem kriminellen, blasphemischen oder amoralischen Sinn, der dem Wort Transgression anhaftet. Der Übertritt bedeutet nicht notwendigerweise eine Grenzverletzung, denn es gibt ritualisierte Formen, die einen bestehenden Limes eher bestätigen als unterminieren oder hinterfragen.

Kulturphilosophische Modelle der Analyse und Konzeptualisierung von Überschreitung/Transgression finden sich etwa bei Georges Bataille (in seiner Ästhetik von Erotik und Gewalt [2004] sowie in seiner Exzess-Ökonomie des

2 Ein in den letzten Jahrzehnten stark erforschter Kulturkomplex, vgl. etwa Derrida, 2001; Bahr, 1994 u. 2005; Hänggi, 2009; Friedrich, Parr, 2009; u. v. a. 
Überschusses [1978]), bei Michel Foucault (s. u.) und in der Denkfigur von De- und Reterritorialisierung bei Gilles Deleuze und Félix Guattari (1974, 252ff.); weiters narratologisch bei den russischen Formalisten (vgl. Ruthner, 2004, 37f.) und Jurij Lotman $(1972)^{3}$ sowie in einer kunsttheoretischen Ausformulierung bei Boris Groys (1992), der in seiner Theorie des Neuen auch das moderne Artefakt eine Grenzüberschreitung zwischen einem profanen und einem quasi-sakralen Raum vollziehen lässt. Aus Platzgründen müssen wir es hier freilich bei einer streiflichtartigen Inspiration durch einige dieser Konzepte belassen.

Da ist zunächst Foucault, der auf das dynamische Verhältnis von Grenze und Überschreitung hingewiesen hat; diese verletzt nicht nur die Grenze, sie redigiert und re-adjustiert sie auch, wenngleich jeder Limes Dauer insinuiert:

Die Überschreitung ist eine Geste, die es mit der Grenze zu tun hat; an dieser schmalen Linie leuchtet der Blitz ihres Übergangs auf [...]. Die Spielregeln der Grenzen und der Überschreitung sind von einer einfachen Hartnäckigkeit: die Überschreitung durchkreuzt immer wieder eine Linie, die sich alsbald in einer gedächtnislosen Woge schließt, um von neuem an den Horizont des Unüberschreitbaren zurückzuweichen. $(2000,31)$

Von einer von Derrida beeinflussten Warte ${ }^{4}$ ließe sich der Hinweis anschließen, wie instabil und in sich widersprüchlich die zentralen Repräsentationsformen unserer Kultur in letzter Konsequenz sind. Ist es diese Unabschließbarkeit der Symbolwelten, ihre wackeligen Grenzen, die bedingen, dass an ihren Rändern und Aporien die Überschreitung wuchern kann als Ausdruck der différance (vgl. Derrida, 2004, insbes. 82)?

Am Beispiel des Gespensternarrativs kann dies näher fokussiert werden: Das Gespenst ist ein durch und durch inzidentielles Wesen einer Transgression; es überschreitet die als äußerst stabil erachtete Grenze zwischen den Räumen des Diesseits und Jenseits in der 'falschen' Richtung und verkörpert damit jene "sich ereignete unerhörte Begebenheit", die Goethe in seine Novellendefinition einschrieb (im Gespräch mit Eckermann, 18. Jan. 1827); daneben hatte das Gespenst nicht zufällig auch auf dem Theater seine Konjunkturen (vgl. Virant, 2013). Es ist die Präsenz von etwas Absentem, Totem; gleichzeitig wiederholt es ein biografisches oder

3 Wenn dieser etwa die Handlung eines literarischen Textes als die Überschreitung eines semantischen Feldes durch (einen) Aktanten fasst.

4 Vgl. Alexander, Smith, 2005, 10: "Derrida (1978) developed a systematic method of reading culture that contextualized structures of discourse and opened them up to creative reconfiguration. Even while affirming the binding influence of already existing representational forms, Derrida insisted on their instability and inevitable productive excess at the margins of meaning. For Derrida, transgression was the shadow of the code, just as for Foucault the cogito must produce and depend upon the 'unthought' [...]." 
historisches Trauma, wie z. B. einen grausamen Mordfall; es verweist auf etwas, das nicht da ist, ein Unbewusstes oder Nicht-Gewusstes, das im kulturellen Gedächtnis erinnert oder verdrängt wird. Der Gespenstertext wiederholt narrativ nun seinerseits diese (unterlassene?) Wiederholung der Erinnerung; zugleich wiederholt er nur die Topik des schon Dagewesenen, d. h. anderer Gespenstergeschichten, das kulturelle Konstrukt dessen, was nicht sein darf - und ist damit zugleich Intertexualität, also auch eine Form kultureller Iteration. Und es wäre nicht vermessen, im Gespenst und in dessen provozierender These von der Alterität des Alltags die Wiederholung der différance und ihrer Irrepräsentabilität zu sehen, ihre wesenlose Spiegelung - so wie die Fantastik, zumal die romantische, ja generell in Spiegel- und andere Identitätsverlustmotive verliebt ist. In den landläufigen Spukgeschichten versucht die Anrufung des Gespenstes und eine damit verbundene Erklärung, diesem final bzw. teleologisch einen Namen zu geben und es damit in die instabile Ordnung des Diskurses gleichsam wieder hineinzuzwingen - ob es gelingt, die Irritation aufzuheben, die Spur, welche die Alterität gelegt hat, ist eine andere Frage. Wie bereits angedeutet wurde, lässt sich nun mit dem Moment der Überschreitung noch jene weitere Denkfigur kombinieren, die aus einer anthropologischen Raumvorstellung stammt: denn was hier produziert wird, ist zumindest für einen Augenblick ein Dazwischen.

\section{Liminalität}

Das vorzustellende Modell wurde in den 1960er Jahren von Victor Turner in Anschluss an Arnold van Genneps Impuls gebendes Werk Rites des passages aus dem Jahr 1909 entwickelt. Liminalität bezeichnet hier ein Übergangsstadium, in dem sich Individuen oder Gruppen befinden, nachdem sie sich rituell von der herrschenden Sozialordnung gelöst haben. Beispiele dafür sind bei Turner die Initiationsriten vorindustrieller Gesellschaften, die auch häufig auf räumlicher Absonderung basieren und die er mit dem Ablaufmodell seines Vorläufers zu beschreiben sucht:

Van Gennep hat gezeigt, daß alle Übergangsriten drei Phasen aufweisen: die Trennungs-, die Schwellen- und die Angliederungsphase. In der ersten Phase (der Trennung) verweist symbolisches Verhalten auf die Loslösung eines Einzelnen oder einer Gruppe von einem früheren fixierten Punkt der Sozialstruktur, von einer Reihe kultureller Bedingungen (einem 'Zustand') oder von beidem gleichzeitig. In der mittleren 'Schwellenphase' ist das rituelle Subjekt (der 'Passierende') von Ambiguität gekennzeichnet; es durchschreitet einen kulturellen Bereich, der wenig oder keine Merkmale des vergangenen oder künftigen Zustands aufweist. In der dritten Phase (der Angliederung und Wiedereingliederung) ist der Übergang vollzogen. Das rituelle Subjekt - ob Individuum oder Kollektiv - befindet sich wieder in einem relativ 
stabilen Zustand und hat demzufolge anderen gegenüber klar definierte, sozialstrukturbedingte Rechte und Pflichten. (Turner, 2005, 94f.)

Liminalität im engeren Sinn findet also in der zweiten Phase statt, jenem "Schwellenzustand", während dessen sich die Individuen in einer ambivalenten Situation befinden: Das Klassifikationssystem der (alltäglichen) Sozialstruktur scheint aufgehoben, die Probanden sind 'betwixt and between', in einen neuen, 'anderen' Zustand versetzt. In diesem liminalen Stadium des Übergangs werden Symbole und Rituale angewendet, um eine gewisse Sicherheit angesichts der hier herrschenden Ungewissheit herzustellen. Durch das Ritual entsteht unter den Teilnehmer(inne)n, die gemeinsam als Gruppe die Schwellenphase durchlaufen, eine Gemeinschaft (communitas), die mit Hilfe der Symbole und des tänzerischen und musikalischen Ablaufs eine gemeinsame, neue Identität herstellen soll. Diese kann verfestigt und betont werden, indem sich das Ritual als Ereignis vom Alltag abhebt und eine Gegenwelt erzeugt.

Im Abschluss werden die Initianden wieder in die soziale Ordnung eingegliedert, aber es ist auch ein endgültiger Bruch mit ihr denkbar. Turner hat später (1989) Van Genneps dreistufiges Ablaufmodell zum viergliedrigen Schema des sog. Sozialen Dramas weiterentwickelt:

I. Bruch mit der sozialen Norm

II. Krise und Konflikt

III. Versuch der Konfliktlösung (Ritual)

IV. Wiedereingliederung oder Abspaltung

Ohne hier auf grundlegende Probleme dieses Ansatzes (etwa im emphatischen Moment der communitas) näher eingehen zu können, wird doch bereits nach dieser kursorischen Skizze klar, dass sich ein redigierter und mit anderem Gedankengut angereicherter Begriff der Liminalität nicht nur für die Fantastik-Theorie als durchaus fruchtbar erweisen könnte.

In actu führt jede Überschreitung zu einem temporären Grenz- oder Zwischenzustand (Liminalität), der gleichsam auf der Demarkationslinie der beiden Felder 'schwebt'. In den plots des Fantastik-Genres wird dies etwa archetypisch in der Figur des Vampirs ausgedrückt, der als Untoter die biologisch-kulturelle Grenze zwischen Tod und Leben (quasi aus der falschen Richtung kommend) aufhebt, weiters jene kartesianische Kluft zwischen Körper und Geist problematisiert und dahinter symbolisch - zumindest im viktorianischen Denken - häufig eine sittliche Grenze zwischen Sexualität und Gewalt verletzt. Diese Liminalität des Vampirs auf der Handlungsebene lässt sich exemplarisch anhand seiner literaturtheoretisch fruchtbaren Definition durch Montague Summers zeigen, die sein dekonstruktives 
Potenzial enthüllt, das hier verräterischerweise mit der cross-gender-Metapher der Androgynität belegt wird:

Throughout the whole vast shadowy world of ghosts and demons there is no figure so terrible, so dreaded and abhorred, yet endowed with such fearful fascination as the vampire; who is himself neither ghost nor demon but who partakes of the dark natures and possesses the mysterious and terrible qualities of both. [...] The vampire has a body and his craving for blood is to obtain sustenance for that body. He is neither dead nor alive; but living in death. He is an abnormality; the androgyne of the phantom world; a pariah among the fiends. (Summers, 1928/61, 1)

Mit der instabilen literarischen Ontologie des Vampirs als Überschreitungsfigur (vgl. Cohen, 1996) korrespondiert auch seine interpretatorische Unfixierbarkeit, seine “Polyvalenz" (Pütz, 1992, 148) als Motiv; H. R. Brittnacher schreibt dazu:

Da Gewalttätigkeit und Blutdurst im Vampirmotiv buchstäblich aus dem Nichts kommen, dient sich seine literarische Adaption grundsätzlich jeder beliebigen Kritik an: Der Vampir erscheint mal als Sinnbild einer entmachteten und rachsüchtigen Aristokratie, mal als Symbol nymphomanischer Weiblichkeit, mal als das eines maßlosen Don-Juanismus, mal wird mit ihm der Stalinismus gebrandmarkt, mal das Franco-Regime, mal die Jesuiten, dann wieder sind es Bürokratie, venerische Krankheiten oder die Furcht vor neueren wissenschaftlichen Entdeckungen wie Hypnose und Magnetismus, die im Vampir ihr Bild gefunden haben. Eben diese Elastizität verbietet eine [...] simple Deutung. $(1994,125)$

In der hier vorgeschlagenen Lesart rührt diese eigentümliche Offenheit des Vampirmotivs von seiner ontologischen - wie narrativen - Liminalität, die Unschlüssigkeit auslöst und Autoren wie Leser zur semantischen Auffüllung jener Leerstellen zwingt, die der untote Blutsauger letztlich be-deutet. Der liminale Raum des Vampirs ist im Grab symbolisiert, das er nach Belieben verlassen kann: wie wir wissen ein eher unmögliches Unterfangen.

\section{Grenzwertigkeiten}

Liminalität scheint also jener Zustand zu sein, in den die Protagonisten fantastischer Texte (und mit ihnen die Leser) angesichts einer 'unerhörten', d. h. potenziell überoder 'un'natürlichen Begebenheit versetzt werden - wenngleich zunächst nicht im Rahmen eines geregelten Rituals, sondern eher eines Zwischenfalls. Die Liminalität ist aber auch gleichsam der Lebensraum, den die fiktiven Monstren (vgl. Cohen, 1996) und andere unerhörte Begebenheiten der Horrorliteratur bewohnen und von hier aus 
die scheinbar eingegrenzte Welt der Normalität heimsuchen; oder z. B. der Raum, in dem schockhaft Metamorphosen (vgl. Reber, 2009; Dorschel, 2009) stattfinden und sich ein Prozess des Werdens zeigt, der weder das Eine noch das Andere ist. Dies lässt sich durchaus mit post/strukturalistischen Modellen im oben angedeuteten Sinne synthetisieren, die in der Fantastik einen Simulationsraum für die De(kon) struktion kultureller Leitdifferenzen - lebend/tot, das Eigene/das Andere etc. - sehen und dahinter in letzter Konsequenz für die Auseinandersetzung mit Alterität und différance. Eine liminale Existenz wie der Vampir, aber auch Kafkas Ungeziefer aus der Verwandlung oder der eingangs erwähnte Odradek entziehen sich ontologischen Kategorisierungen:

Die einen sagen, das Wort Odradek stamme aus dem Slawischen und sie suchen auf Grund dessen die Bildung des Wortes nachzuweisen. Andere wieder meinen, es stamme aus dem Deutschen, vom Slawischen sei es nur beeinflußt. Die Unsicherheit beider Deutungen aber läßt wohl mit Recht darauf schließen, daß keine zutrifft, zumal man auch mit keiner von ihnen einen Sinn des Wortes finden kann. (Kafka, 1994, 222)

Prinzipiell ist also Liminalität auf mehreren Ebenen vorstellbar:

a. räumlich/geografisch: als Zwischenraum

b. persönlich/psychisch: als Zwischenzustand

c. semantisch/narrativ: als Ambivalenz und Uneindeutigkeit.

Anstelle eines Schlusswortes seien deshalb noch einige Fälle aufgelistet, in denen die Denkfigur des Liminalen eine heuristische Rolle spielt bzw. spielen könnte, insofern als sie psychische und semantische Prozesse in eine räumliche Metaphorik übersetzt:

- In einem unveröffentlichten Vortrag hat Wolfgang Müller-Funk Turner (2003) mit Michail Bachtin (1975/2008) zusammengebracht und Chronotopen (Raum-Zeit-Strukturen) des Liminalen bzw. dessen Orte aufgelistet: so z. B. Türen, Brücken, Tunnel, Membranen, aber auch jenes Sitting on a Fence, von dem die Rolling Stones sangen.

- Ein notorischer liminaler Zwischenraum ist Homi Bhabhas “Third Space” seit seiner Emergenz in der postkolonialen Theoriebildung geworden: Es ist jener (utopische) Ort, der in der Begegnung zweier Kulturen bzw. Teilnehmer/ innen entsteht, in dem Bedeutungen neu ausgehandelt werden können (vgl. Babka, Malle, Schmidt, 2012): 
It is that Third Space, though unrepresentable in itself, which constitutes the discursive conditions of enunciation that ensure that the meaning and symbols of culture have no primordial unity or fixity; that even the same signs can be appropriated, translated, rehistoricized and read anew. (Bhabha 1994, 37)

- Liminalität wird damit auch zu einer Parallelaktion zur Begrifflichkeit der Hybridität (vgl. Bhabha, 1994, 38 et passim; Bhabha, 2016): Bedeutet diese aber eine unauflösliche Amalgamierung, so hebt das Liminale eher auf die Behauptung eines Dazwischen, also eines Schwebezustands aber, der auch zwischen Aggregationen bestehen kann, die prinzipiell nicht vermischbar sind.

- Liminalität könnte auch den Area Studies neue Impulse geben, indem sie Zwischenräume besser konzeptualisieren lässt: das Grenzland vielerorts ebenso wie ganze Regionen, die von jeher im Geruch des Heterogenen, der konfrontativen wie auch der friedlichen Angrenzung stehen. Dies ließe sich etwa auf das deutsch-polnisch-tschechische Schlesien ebenso anwenden wie auf größere Teile des europäischen Kontinents, wie z. B. den sog. Balkan (vgl. Todorova, 2005) oder 'Mittel-' bzw. 'Zentral'europa (vgl. Ruthner, 2017).

- Liminalität gibt es auch als epistemologisches Phänomen von Wissenskulturen. Als sich etwa in den ersten Jahren des 20. Jahrhunderts die freudianische Einsicht durchzusetzen begann, dass die Trennung zwischen Kindheit und Erwachsensein nicht durch das Fehlen bzw. von Vorhandensein von Sexualität definiert werden kann, führte diese neue Grauzone förmlich zu einer liminalen Verstörung einer ganzen Kultur, die sich in den gewalttätigen Kindfrau-Fantasien Wedekinds, aber auch dem scheinbar hilarischen Mutzenbacher-Roman von 1906 Bahn bricht (vgl. etwa Ruthner, Schmidt, 2019, 21f.).

- Liminalität als Thema folgt nicht nur einem quasi anthropologischen Interesse, sondern partizipiert auch an der Kulturindustrie der Moderne und ihren Märkten, denen sie Material für ihre Innovationsbewegungen die letztlich genauso Grenzüberschreitungen sind - zur Verfügung stellt (vgl. Ruthner, 2004, Kap. 1). Durch ihre kapitalistischen Produktionsbedingungen, die mit einer Überbietungsästhetik Hand in Hand gehen, sieht sich nämlich die Literatur der Moderne - wie auch andere kulturelle Felder (vgl. Bourdieu, 1999) - gezwungen, permanent gegen ihre Regeln, ihren Kanon zu verstoßen und das Neue zu suchen, z. B. durch die Schaffung 'sub'kultureller Segmente (vgl. Ruthner, 2004, Kap. 1); speziell alle Avantgarde-Bewegungen sind davon betroffen. Es ist also die Grenze, die in unserer Kultur paradoxerweise 
zur Überschreitung und damit zur Liminalität einlädt. ${ }^{5}$ In der liminalen Unentschiedenheit, im Flottieren und Werden entstehen dann häufig neue Grenz-Werte.

\section{Bibliographie}

Achilles, J., u. a. (Hg.), Liminale Anthropologien. Zwischenräume in Literatur und Philosophie, Würzburg 2012.

Aguirre, M., Sutton, Ph., Margins and Thresholds. An Enquiry into the Concept of Liminality in Text Studies, Madrid 2000.

Alexander, J., Smith, Ph. (Hg.): The Cambridge Companion to Durkheim, Cambridge 2005.

Augé, M., Non-Places. An Introduction to Supermodernity, London, New York 2008.

Babka A., Malle, J., Schmidt, M. (Hg.): Dritte Räume. Homi Bhabhas Kulturtheorie. Kritik. Anwendung. Reflexion, Wien, Berlin 2012.

Bachtin, M., Chronotopos [1975], Frankfurt a. M. 2008.

Bahr, H.-D., Die Sprache des Gastes. Eine Metaethik, Leipzig 1994.

Bahr, H.-D., Die Befremdlichkeit des Gastes, Wien 2005.

Bataille, G., Die psychologische Struktur des Faschismus / Die Souveränität, München 1978.

Bataille, G., Die Tränen des Eros, München 2004.

Benjamin, W., Das Passagen-Werk, Bd. 1, Frankfurt a. M. 1983.

Bhabha, H., The Location of Culture, London, New York 1994.

Bhabha, H., Über kulturelle Hybridität. Tradition und Übersetzung, Wien, Berlin 2016.

Bourdieu, P., Die Regeln der Kunst. Genese und Struktur des literarischen Feldes, Frankfurt a. M. 1999.

Brittnacher, H. R., Ästhetik des Horrors. Gespenster, Vampire, Monster, Teufel und künstliche Menschen in der phantastischen Literatur, Frankfurt a. M. 1994.

Cohen, J. J., Monster Theory. Reading Culture, Minneapolis 1996.

Deleuze, G., Guattari, F., Anti-Ödipus. Kapitalismus und Schizophrenie 1 [1972], Frankfurt a. M. 1974.

Derrida, J., Von der Gastfreundschaft, mit einer 'Einladung' von Anne Dufourmantelle (Hg. Engelmann, P.) Wien 2001.

5 Der Kultursoziologe Bernhard Giesen hat aufgezeigt (2010), wie Grenzverletzungen die Ordnung zu adjustieren helfen und Zwischenlagen das System kultureller Differenz nicht untergraben, sondern bestätigen. 
Derrida, J., Die différance, in: Postmoderne und Dekonstruktion. Texte französischer Philosophen der Gegenwart (Hg. Engelmann, P.), Stuttgart 2004.

Donec, P., Die Grenze. Eine konzeptanalytische Skizze der Limologie, Würzburg 2014.

Dorschel, A., Verwandlung. Mythologische Ansichten, technologische Absichten, Göttingen 2009.

Foucault, M., Vorrede zur Überschreitung, in: Von der Subversion des Wissens (Hg. Seitter, W.), Frankfurt a. M. 2000, S. 28-45.

Friedrich, P., Parr, R. (Hg.), Gastlichkeit. Erkundung einer Schwellensituation, Heidelberg 2009.

Geisenhanslüke, A., Mein, G. (Hg.), Schriftkultur und Schwellenkunde, Bielefeld 2008.

Gießen, B., Zwischenlagen. Das Außerordentliche als Grund der sozialen Wirklichkeit, Weilerswist 2010.

Groys, B., Über das Neue. Versuch einer Kulturökonomie, München 1992.

Hänggi, Ch., Gastfreundschaft im Zeitalter der medialen Repräsentation, Wien 2009.

Kafka, F., Die Sorge des Hausvaters, in: Ein Landarzt und andere Drucke zu Lebzeiten (Hg. Koch, H.-G.), Frankfurt a. M. 1994, S. 222-223.

Klapcsik, S., Liminality in Fantastic Fiction. A Poststructuralist Approach, Jefferson, London 2012.

Krüger, K., Bildpräsenz - Heilspräsenz. Ästhetik der Liminalität, Göttingen 2018.

Lachmayer, H., Plica, P. (Hg.), Über die Schwelle, Wien 2003.

Lotman, J., Die Struktur literarischer Texte, München 1972.

Magris, F., Die Grenze. Von der Durchlässigkeit eines trennenden Begriffs, Wien 2019.

Müller-Funk, W., The Poetics of Spaces in-between (unveröff. Vortrag am Trinity College Dublin) 2013.

Pütz, S., Vampire und ihre Opfer. Der Blutsauger als literarische Figur, Bielefeld 1992.

Reber, U., Formenverschleifung. Zu einer Theorie der Metamorphose, München 2009.

Rosello, M., Wolfe, St., Introduction, in: Border Aesthetics. Concepts and Intersections (Hg. Wolfe, St., Schimanski, J.), New York, Oxford 2017, S. 1-20.

Ruthner, C., Am Rande. Kanon, Kulturökomonie und Intertextualität des Marginalen am Beispiel der (österreichischen) Phantastik im 20. Jh., Tübingen, Basel 2004.

Ruthner, C., Zur Theorie der Liminalität, oder: Die Grenzwertigkeit der Fantastik, in: Der Schauer(roman). Formen - Diskurszusammenhänge - Funktionen (Hg. Grizelj, M.), Würzburg 2010, S. 77-90. 
Ruthner, C., Fantastic Liminality. A Theory Sketch, in: Collisions of Reality. Establishing Research on the Fantastic in Europe (Hg. Schmeink, L., Böger, A.), Berlin, New York 2012, S. 35-49.

Ruthner, C., Collateral Roadkill. The Conflicted Death of "Central Europe" en Route to Sarajevo and Brussels, in: Narrative(s) in Conflict (Hg. Ruthner, C., Müller-Funk, W.), Berlin, Boston 2017, S. 165-186.

Ruthner, C., Schmidt, M. (Hg.), Die Mutzenbacher. Kontexte und Lektüren eines Skandalromans, Wien 2019.

Simmel, G., Soziologie des Raumes, in: Aufsätze und Abhandlungen 1901-1908. Bd. 1, Frankfurt a. M. 1995, S. 132-183.

Summers, M., The Vampire. Its Kith and Kin, London 1928, reprint New York 1961.

Todorova, M., Spacing Europe. What is a Region? East-Central Europe 32, 2005, H. $1-2$, S. 59-78.

Turner, V., Das Ritual. Struktur und Anti-Struktur [1969], Frankfurt a. M., New York 2005.

Turner, V., Vom Ritual zum Theater. Der Ernst des menschlichen Spiels, Frankfurt a. M. 1989.

Virant, Š., Wanderungen der Untoten, in: Literatur am Rand. Perspektiven der Trivialliteratur vom Mittelalter bis zum 21. Jahrhundert (Hg. Parra Membrives, E., Classen, A.), Tübingen 2013, S. 269-277. 


\section{Clemens Ruthner \\ Mejno in vmesno. Liminalnost kot miselni prostor}

Ključne besede: kulturna teorija, kulturologija, literarna veda, liminalnost, literarna antropologija, meja, transgresija, duhovi, vampirji, tretji prostor, prag

Članek obravnava koncept »liminalnosti«, ki sta ga prva formulirala antropologa Arnold van Gennep in Victor Turner. »Liminalnost« je prostorska miselna figura, ki raziskuje (družbene) medprostore in mentalna stanja, kot so ritualni pragovi in drugi prostori prehajanja, iniciacijski rituali in podobno. Povezana je s temeljnimi antropološkimi pojmi, kot sta prag (meja ali omejitev, ki označuje teritorialnost človeškega življenja) in transgresija, pa tudi s »tretjim prostorom « Homija Bhabhe. Liminalnost udejanja (utopično?) umetnost postajanja, ki presega ali premosti dihotomije. Po drugi strani pa lahko pomeni tudi ujetost med kulturne, družbene in diskurzivne fronte, in sicer na precej katastrofičen način (kot na primer $\mathrm{v}$ Preobrazbi Franza Kafke). Članek, ki uvaja večji raziskovalni projekt, oriše različne vidike »liminalnosti«, nato pa predlaga možne aplikacije na področju literarnih ved in kulturologije, na primer $\mathrm{v}$ raziskavah motivov duhov, prikazni in vampirjev $\mathrm{v}$ literarni fantastiki ali pri študijah regij (Balkan ali Srednja Evropa kot mejni prostori kultur in politike). 


\section{Clemens Ruthner \\ Borderline, in-between. Liminality as Thought Space}

Keywords: cultural theory, cultural studies, literary studies, liminality, literary anthropology, borders and boundaries, transgression, ghosts, vampires, third space, thresholds

This article presents and discusses the concept of "liminality", which was first formulated by the anthropologists Arnold van Gennep and Victor Turner. The "liminal" is a spatial figure of thought that explores in-between (social) spaces or states of mind, such as ritual thresholds and other places of transit, rites of passage, etc. It is connected to basic anthropological concepts such as the limen (borders, boundaries and limits demarcating the territoriality of human life), or transgression, and it is also related to Homi Bhabha's "Third Space". The liminal practices a (utopian?) art of becoming that claims to bypass or bridge dichotomies; on the other hand, it can also mean to be caught between cultural, social, and/or discursive frontlines in a rather catastrophic way (such as in Kafka's Metamorphosis). The article, which is a first step into a bigger project, sketches some of these facets of "liminality" before proposing and investigating potential applications in the fields of literary and cultural studies: e.g. when it comes to the ghost apparitions and vampire figures of the literary fantastic, or to Area Studies (the Balkans and Central Europe as liminal spaces of culture/s and politics). 